\section{MJFF's progress in markers}

\section{By Michael J. Haas, Senior Writer}

The Michael J. Fox Foundation for Parkinson's Research has taken two major steps toward its goal of identifying clinical populations in which new therapies for Parkinson's disease can be tested. Last week, the foundation made available the first set of data and samples from its LRRK2 Cohort Consortium and presented preliminary data from its Parkinson's Progression Marker Initiative.

Now, the LRRK2 Cohort Consortium will conduct longitudinal studies in its enrolled participants, whereas the Parkinson's Progression Marker Initiative (PPMI) aims to confirm the preliminary findings in the full study cohort. Additionally, the PPMI is planning collaborations with the Alzheimer's Association's Alzheimer's Disease Neuroimaging Initiative (ADNI).

The LRRK2 story dates to 2004, when two independent teams identified mutations in leucine-rich repeat kinase 2 (LRRK2) in patients who had an inherited form of PD. "That discovery galvanized a lot of industry interest because LRRK2 is a protein kinase with gainof-function mutations in PD, and so it seemed a tractable target for inhibition," Todd Sherer, CEO of MJFF, told SciBX.

In parallel with industry efforts to develop LRRK2 inhibitors, MJFF sought "to get in front of the curve by developing a cohort of individuals with LRRK2 mutations that would help establish the infrastructure for later clinical and biomarker research," he said.

Thus, MJFF launched the LRRK2 Cohort Consortium in 2009 with a pilot program that included two populations-Ashkenazi Jews and North African Berbers-in whom LRRK2 mutations account for about $20 \%$ and $40 \%$ of PD cases, respectively.

"We brought together the researchers at centers in New York, Tel Aviv and Tunisia with a shared core protocol" for cross-sectional studies in patients with $\mathrm{PD}$, healthy individuals who harbored the LRRK2 mutations and healthy individuals who did not, Sherer said.

In 2011, MJFF expanded the consortium to include a total of about 1,900 individuals at clinical centers in 9 countries, including Norway, Spain, Germany and Canada.

Sherer said the purpose of the cross-sectional studies was twofold. One goal was to compare LRRK2 PD and idiopathic PD. The other was to pre-recruit a population of individuals carrying LRRK2 mutations for future clinical trials, "so that the trials don't have to start recruiting from scratch and there will be some run-in data for the population," he said.

Now that the consortium has completed those cross-sectional studies, MJFF has made the clinical data and biological samples-including whole blood, serum, urine and cerebrospinal fluid (CSF) - available to academic and industry researchers for the first time.

Although any qualified researcher can gain access to the clinical data, "we will set a higher bar of acceptance for proposed projects that would use the biological samples because they are a limited resource," Sherer said. For example, "we would want to see data to confirm the reliability and validity of an assay that would use the samples."

He added, "We are especially interested in projects involving pharmacodynamic measurements of LRRK2 levels and activity in the samples that could be used to measure outcomes in trials of LRRK2related therapies."

PD researchers in academia and industry can apply for access to the consortium's data and specimens by submitting project proposals. MJFF will review new proposals and decide whether to accept them only in August, October and December.

"We set the deadlines to encourage researchers to apply in a timely manner," Sherer said.

Academic and industry researchers can also apply to MJFF for up to $\$ 250,000$ in funding for projects that use data or samples from the LRRK2 Cohort Consortium.

Meanwhile, all but one of the groups within the consortium is converting the crosssectional studies to longitudinal studies. "The Tunisian group is not lined up to continue due to the geopolitical situation in the country," Sherer said. "It's not clear to us how we could continue that study_but this doesn't mean it will never continue."

MJFF expects to make preliminary data from the longitudinal studies available in 2014 "rather than waiting for the studies to finish in late 2018 or early 2019."

To date MJFF has invested more than $\$ 10$ million in the LRRK2 Cohort Consortium. The clinical centers have also invested in the consortium.

"We see the LRRK2 Cohort Consortium as an example of the exact role the foundation can play because the findings are a resource that everyone in industry is looking for but will not necessarily have funded in house," Sherer said.

\section{PD and AD overlap}

MJFF also announced the availability of data and samples from its PPMI, a five-year program launched in 2010 to identify biological markers for PD.

The program initially enrolled about 150 patients and age-matched controls in the U.S. and $\mathrm{EU}^{1}$ and since has expanded to include about 600 patients and controls at 24 sites in the U.S., EU and Australia. 
According to Mark Frasier, MJFF's VP of research programs, the PPMI program is examining the levels of four proteins in CSF: $\alpha$-synuclein (SNCA), $\beta$-amyloid (A $\beta$ ), microtubule-associated protein- $\tau$ (MAPT; TAU; FTDP-17) and phosphorylated- $\tau$ (p- $\tau$ ).

"In the preliminary data from the first 100 participants who enrolled in PPMI, we've found that the levels of all four proteins are lower in the CSF of PD patients than in the healthy controls," Frasier said.

Additionally, the preliminary data showed differences in the levels of the markers between subgroups of patients with PD whose clinical symptoms differ, he said. "For example, in patients who experience significant problems with posture and gait, the levels of all four markers are lower than in patients who experience significant problems with tremors."

These findings suggest the CSF markers could be used to stratify patients in clinical trials of therapies to treat symptoms associated with particular subtypes of PD.

Frasier said PPMI researchers also compared the preliminary data with those from the Alzheimer Association's ADNI program, which is examining the same four CSF markers in patients with AD and healthy controls, and found some interesting overlaps.

Indeed, MJFF's interest in markers extends beyond LRRK2 and even beyond PD itself.

For example, in patients with $\mathrm{AD}, \mathrm{A} \beta$ is also lower but TAU is higher than levels in controls, he said. "The findings point to a potential role for TAU in both PD and AD, though we don't yet know TAU's role in memory impairment in $\mathrm{AD}$ or its role in PD symptoms."

Kenneth Marek presented the preliminary PPMI data and the comparisons with the ADNI results at the Alzheimer's Association International Conference last week. Marek is a clinical professor of neurology at Yale University and president and senior scientist at the university's Institute for Neurodegenerative Disorders.

Frasier said a paper reporting the preliminary data is in the press.
Going forward, PPMI researchers will analyze the markers in the full cohort of 600 patients with PD and controls to confirm the preliminary findings, Frasier said.

"Also, we will examine how these markers change over time by comparing samples taken at six months and one year with the baseline samples and see how levels of the markers might change as the disease progresses," he said.

Frasier added that PPMI and ADNI plan to use data from both programs to investigate questions about aging and neurodegeneration and want to add new parameters or studies to each program.

"There is momentum among researchers in both $\mathrm{PD}$ and $\mathrm{AD}$ to test drugs earlier in the disease process-before overt symptoms appearbecause we think the greatest chance for therapeutic success occurs when the loss of brain cells is minimal," which, in turn, underscores the need to identify the right population and methods for clinical trials, he said.

The markers studied in the PPMI and ADNI programs "could help identify the presymptomatic population for testing new therapies in the clinic," said Frasier. "Both Parkinson's disease and Alzheimer's disease research is moving in this direction."

MJFF plans to disclose details about the PPMI-ADNI collaborations in the coming months. To date MJFF has invested \$55 million in the PPMI program.

Haas, M.J. SciBX 6(28); doi:10.1038/scibx.2013.710

Published online July 25, 2013

\section{REFERENCES}

1. Haas, M.J. SciBX 4(29); doi:10.1038/scibx.2011.813

\section{COMPANIES AND INSTITUTIONS MENTIONED}

Alzheimer's Association, Chicago, III. The Michael J. Fox Foundation for Parkinson's Research, New York, N.Y.

Yale University, New Haven, Conn. 